\title{
An on-Line Extraction Method for the Parasitic Capacitance of the Photovoltaic Panel
}

\author{
Jianing Wang, Yuanwu Xun, Xiaohui Liu, and Shaolin Yu
}

\begin{abstract}
In the photovoltaic (PV) plant, the parasitic capacitance between the PV panel and the ground $\left(C_{\mathrm{PV}}\right)$ causes leakage current in the non-isolated systems. The case can be deteriorated in the rainy environment because the $C_{\mathrm{PV}}$ increases dramatically due to the rainwater. The existing off-line methods are either through simulation or measurement by impedance analyzer, which are not easy to conduct on-site especially in the PV plant when it rains. The existing on-line capacitance measurement method is to measure the amplitude of the main components of voltage and current on the capacitance, but the measurement results are inaccurate because the parasitic resistance and inductance of the PV panels are not considered. This paper presents a simple on-line extraction method for the $C_{\mathrm{PV}}$, which is obtained through measurement of the oscillation period of the leakage current. In this way, the $C_{\mathrm{PV}}$ can be obtained even the equipment is running. The principle is analyzed in a full-bridge case and the simulation and experiment are carried out for the verification. Finally, the $C_{\mathrm{PV}}$ obtained in this method is compared with the 3D finite element simulation, the measurement by impedance analyzer and the existing on-line calculation method, which shows the accuracy of this proposed method.
\end{abstract}

Index Terms - Leakage current, parasitic capacitance, photovoItaic panel, oscillation.

\section{INTRODUCTION}

$\mathrm{T}$ HE parasitic capacitances between the photovoltaic (PV) panel and the ground $\left(C_{\mathrm{PV}}\right)$ can be regarded as the sum of the three parts: cell-to-frame capacitance $C_{\text {cf }}$, cell-torack capacitance $C_{\mathrm{cr}}$ and cell-to-ground capacitance $C_{\mathrm{cg}}$ [1][4]. Other parasitic parameters of the PV panel, such as the equivalent inductance and resistance of the frame, rack and cable $L_{\mathrm{e}}$ and $R_{\mathrm{e}}$ [2]. As shown in Fig. 1(a). The $C_{\mathrm{PV}}$ depends on the installation method and the size of the panel [5]-[10]. Meanwhile, the rainwater as an electrode or dielectric can change the capacitances, sometimes dramatically [2]-[4]. In normal environment, the $C_{\mathrm{PV}}$ can vary from $7 \mathrm{nF} / \mathrm{kW}$ [11]-[12]

Manuscript received July 20, 2019; revised September 4, 2019; accepted November 27, 2019. Date of publication December 30, 2019; date of current version November 30,2019 . This work is supported by National Natural Science Foundation of China (51607053) and Sponsored by grants from the Power Electronics Science and Education Development Program of Delta Group. This paper was presented in part at the 2019 IEEE 10th International Symposium on Power Electronics for Distributed Generation Systems (PEDG), Xi'an, China, June 2019.

All authors are with the Department of Electrical Engineering and Automation, Hefei University of Technology, Hefei 230009, China (e-mail: 16002530@163.com; 1402900164@qq.com; 497676042@qq.com; hajcysl@163.com).

Digital Object Identifier 10.24295/CPSSTPEA.2019.00030

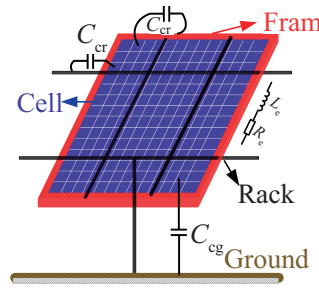

(a) (b)

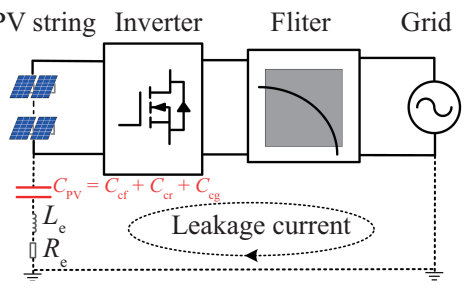

Fig. 1. (a) Distributed parasitic capacitance model of PV panel. (b) Simplified common mode leakage current in a PV transformerless inverter.

to $220 \mathrm{nF} / \mathrm{kW}$ [13]-[14]. However, it can even reach $1 \mu \mathrm{F} / \mathrm{kW}$ in damp environment [2], [15]. In non-isolated systems, the parasitic capacitance $C_{\mathrm{PV}}$ causes common-mode (CM) current [5], [8], [16], as shown in Fig.1(b). The CM current is sensitive to the $C_{\mathrm{PV}}$, so it is crucial to extract the capacitance value accurately [2]-[5].

There are many researches on the extraction of this capacitance, the methods can be mainly characterized into four categories: analytical method [2], [11], [17]-[19]; numerical simulation [1]-[4], [20]; direct measurement method [4], [21] and indirect measurement method [7], [9], [22], [23].

The analytical method is to calculate the capacitance of the object based on the existing theoretical formulas. It is necessary to measure the shape and size of the PV panel, then calculate the capacitance according to the obtained data. But in the reality, the size of PV panels is complex and irregular that causes difficulty measuring [2]. In an ideal environment, the calculation process is relatively simple, but it will become very complicated when considering the actual factors such as edge effect.

By using the finite element (FE) analysis software such as Ansys Maxwell, the manual complicated operations can be avoided, so the numerical simulation method is proposed [2][4]. But the accurate results of this method still depend on accurate measurement and modeling. When the model contains too many factors such as the actual environment, it will lead to a time-consuming simulation.

Compared to the methods of analytical and numerical calculation, the direct measurement method will achieve accurate results with no consideration of factors such as size and environment. This method uses the test equipment such as impedance analyzer to get the measurement results directly. However, when many PV panels are connected in series, the high voltage of the ports will make the measurement process unsafe. Furthermore, this is an off-line measurement 
method, which is hard to operate on-site especially when the environmental factors change.

Thus, the indirect method can be applied to get the parasitic capacitance by measuring other electrical parameters on-line in the circuit. [7], [9] calculates the $C_{\mathrm{PV}}$ based on the amplitude of main components of voltage and current on the capacitance. According to Ohm's law, a series of capacitance values can be calculated under a series of voltage harmonics and current harmonic components. Then $C_{\mathrm{PV}}$ obtained by average of the series of capacitances. This is an on-line method to obtain the parasitic capacitance value, but it requires simultaneous measurement the amplitude of main components of voltage and current on the capacitance and does not consider the effects of parasitic resistance and inductance of the PV panels such as $L_{\mathrm{e}}$ and $R_{\mathrm{e}}$. This will cause complex test operations and inaccurate results.

In order to solve the problems above, this paper proposes a simple on-line $C_{\mathrm{PV}}$ extraction method, which not only overcomes the shortcomings of off-line methods but also ensures the accuracy of the results. Firstly, by analyzing each switching process of single-phase full-bridge inverter circuit, a unified resonant circuit model is obtained. Secondly, according to the model, the relationship between the $C_{\mathrm{pv}}$ and the leakage current oscillation period $T$ is derived. Then, simulations and experiments are presented below. Finally, the parasitic capacitance obtained in this proposed method is compared with the 3D FE simulation, the measurement by impedance analyzer and the existing on-line calculation method, which shows the accuracy of this method.

\section{Photovoltaic Panel Parasitic Capacitance EXTRACTION METHOD}

The single-phase full-bridge non-isolated inverter is chosen as an example for analysis in this paper, as shown in Fig. 2 The PV is a series of photovoltaic panels. $C_{\mathrm{DC}}$ is the DC side support capacitance that usually is a Millifarad level capacitance. $S_{1} \sim S_{4}$ are the switches that form the full bridge. $L_{1}$ and $L_{2}$ are the filter inductance. $C$ is the filter capacitance that is usually above 10 $\mu \mathrm{F} . R$ is a resistance load. $R_{\mathrm{g}}$ represents the resistance between load ground and chassis of the inverter. The $V_{\mathrm{DC}}$ is the voltage of the $C_{\mathrm{DC}}$, which is considered as a stable voltage source. The $v_{\mathrm{Cpv}}$ is the voltage of the $C_{\mathrm{PV}}$, and its changes will lead to a leakage current $i_{\text {leak }}$ [15]. The points 1 and 2 represent the outputs of bridges respectively, $a$ and $b$ are the two ends of the $\mathrm{PV}$ and the ground.

In Fig. $2, L_{\text {bus } 1}, L_{\text {bus } 2}, L_{\text {bus } 3}$ and $L_{\text {bus } 4}$ are stray line inductances [5]. $C_{\mathrm{p} 1}, C_{\mathrm{p} 2}, C_{\mathrm{p} 3}$, and $C_{\mathrm{p} 4}$ are the parasitic capacitances between the switch-to-heatsink/ground [24]. Due to the $C_{\mathrm{PV}}$ in a PV plant is much larger than the switch-to-heatsink/ground capacitances [24], [25], and the switch-to-heatsink/ground parasitic capacitances are connected in parallel with $C_{\mathrm{PV}}$, so the capacitance of the switches can be ignored. The inductance of the filter inductor is much larger than the stray inductances of the line [5], and the stray inductances are connected in series with the filter inductances, so the stray inductance of the line

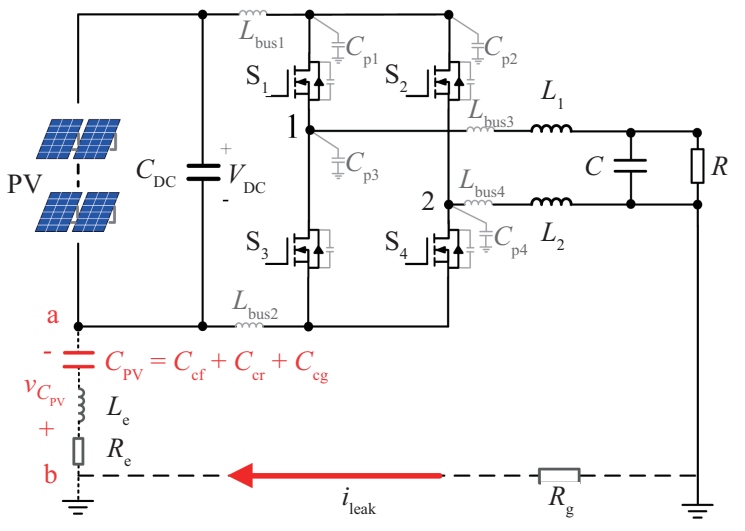

Fig. 2. Single-phase full-bridge non-isolated inverter with parasitics.

TABLE I

Switching State of Unipolar \& Frequency Multiplication SPWM Strategy

\begin{tabular}{cccccc}
\hline \hline State & Output & $\mathrm{S}_{1}$ & $\mathrm{~S}_{2}$ & $\mathrm{~S}_{3}$ & $\mathrm{~S}_{4}$ \\
\hline State1 & $\mathrm{P}$ & $\mathrm{ON}$ & OFF & OFF & ON \\
State2 & O1 & ON & ON & OFF & OFF \\
State3 & O2 & OFF & OFF & ON & ON \\
State4 & $\mathrm{N}$ & OFF & ON & ON & OFF \\
\hline
\end{tabular}

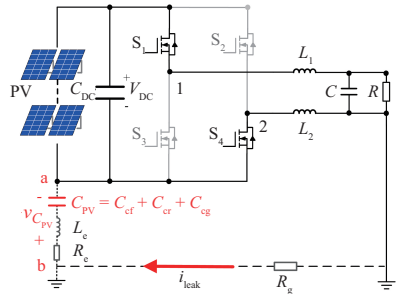

(a)

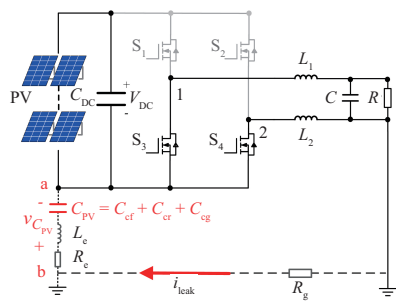

(c)

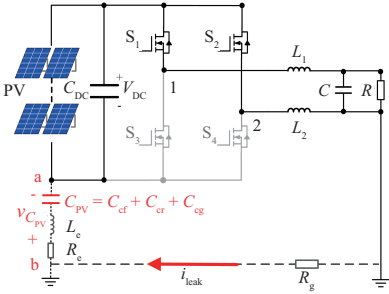

(b)

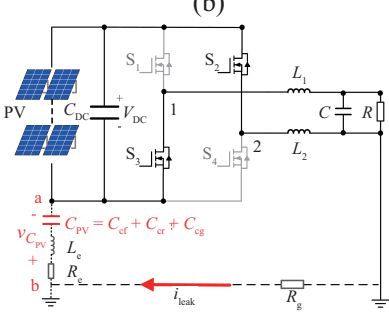

(d)
Fig. 3. The states of single-phase full-bridge circuit under the unipolar \& frequency multiplication SPWM. (a) State 1. (b) State 2. (c) State 3. (d) State 4.

can be ignored.

When using the unipolar \& frequency multiplication sinusoidal pulse width modulation (SPWM), the four switch states and output modes are shown in Table I. In the positive half cycle, the output of bridge switches between state 1 and state 2, or state 1 and state 3 . And in the negative half cycle, the output is between state 2 and state 4 , or state 3 and state 4 . Next, the above switching processes will be analyzed respectively.

The circuit in these four states is shown in Fig. 3. The switch can be considered as short when it is active, the considered 


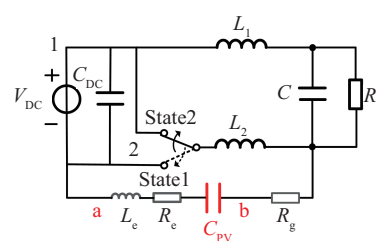

(a)

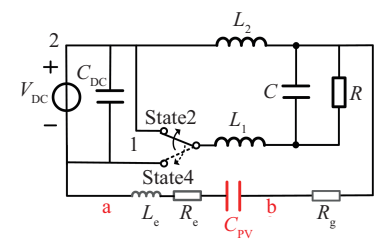

(c)

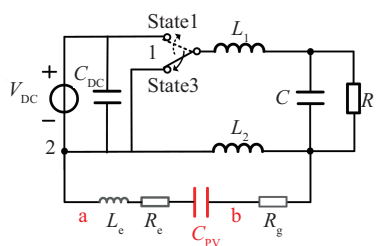

(b)

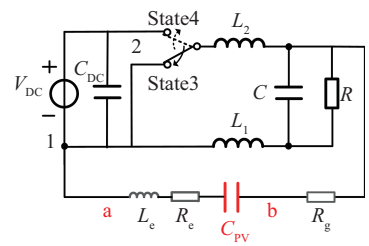

(d)
Fig. 4. The simplified models of switching processes. (a) State 1 to state 2 (state 2 to state 1). (b) State 1 to state 3 (state 3 to state 1). (c) State 4 to state 2 (state 2 to state 4 ). (d) State 4 to state 3 (state 3 to state 4 ).

as open in other conditions. Based on these assumptions, the switching processes discussed above can be simplified as shown in Fig. 4.

The simplified models of switching processes are shown in Fig. 4, which the dotted line indicates the processes in the brackets. Then the models can be classified into the following four models. The model after switching from state 2 to state 1 (or state 3 to state 1) is shown in Fig. 5(a); the model after the switching from state 1 to state 2 (or state 4 to state 2 ) is shown in Fig. 5(b); the model after the switching from state 1 to state 3 (or state 4 to state 3 ) is shown in Fig. 5(c); the model after the switching from state 2 to state 4 (or state 3 to state 4 ) is shown in Fig. 5(d).

The oscillation period of the high-order circuit is only related to its structure, therefore the source can be ignored because it does not affect the resonant period in the circuit. Meanwhile, due to the support capacitor $C_{\mathrm{DC}}$ and the filter capacitance $C$ are very large, they can be regarded as a short circuit in a high frequency environment. Then the classified models are normalized to a final model that is shown in Fig. 6.

From the final model, it is known that:

$$
f_{\mathrm{r}}=\frac{1}{2 \pi \sqrt{L_{\mathrm{eq}} \times C_{\mathrm{PV}}}}
$$

The $f_{\mathrm{r}}$ is the oscillation frequency of the equivalent resonant circuit model. The $L_{\text {eq }}$ is the equivalent inductance of this equivalent resonant circuit model. The $L_{\mathrm{e}}$ inductance of the PV frame, rack and cable is much smaller than the filter inductor [2], and this inductance is connected in series with the filter inductances, so $L_{\mathrm{e}}$ can be ignored.

In Fig. 6(b), the $L_{\mathrm{eq}}$ is:

$$
L_{\text {eq }}=\frac{L_{1} \times L_{2}}{L_{1}+L_{2}}
$$

The $R_{\text {eq }}$ is the equivalent resistance of this equivalent

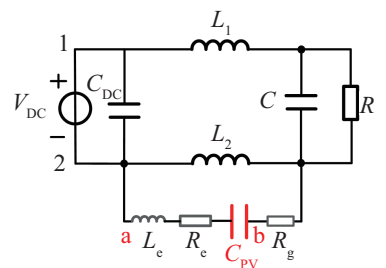

(a)

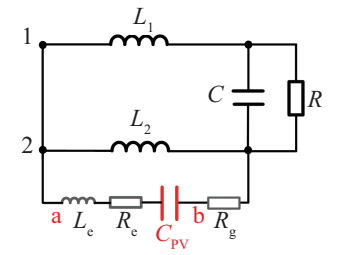

(c)

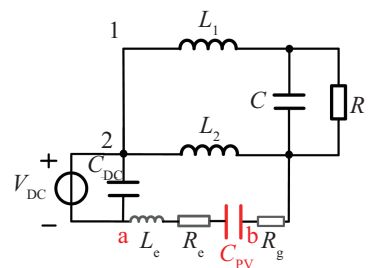

(b)

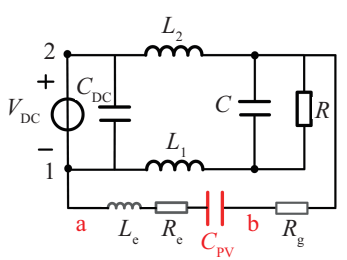

(d)
Fig. 5. The classified models of the switching processes. (a) State 2 to state 1 (state 3 to state 1). (b) State 1 to state 2 (state 4 to state 2). (c) State 1 to state 3 (state 4 to state 3 ). (d) State 2 to state 4 (state 3 to state 4 ).

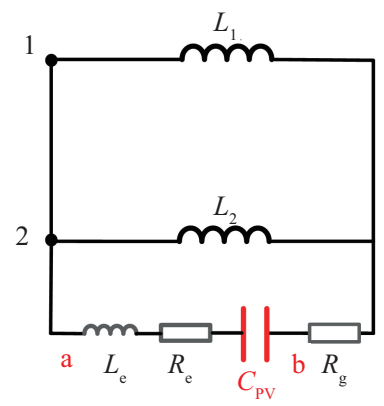

(a)

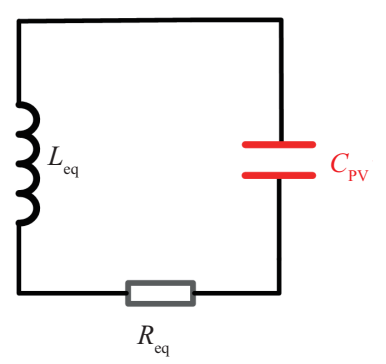

(b)
Fig. 6. (a) Equivalent resonant circuit model in each switching states. (b) Final equivalent resonant circuit model.

resonant circuit model, but $R_{\text {eq }}$ has no effect on the resonant frequency and only affects the amplitude of the oscillation. Therefore, the $C_{\mathrm{PV}}$ extraction method proposed in this paper is more accurate than the existing online methods.

Since the impedance at the resonance frequency point is the smallest, there is a large current at the oscillation frequency of the equivalent resonance circuit, so the oscillation frequency of the leakage current is the oscillation frequency of the equivalent circuit.

The relationship between the parasitic capacitance $C_{\mathrm{PV}}$ and the oscillation period of the leakage current $T$ is:

$$
C_{\mathrm{PV}}=\frac{L_{1}+L_{2}}{4 \pi^{2} \times L_{1} \times L_{2}} \times T^{2}
$$

Through (3), when the leakage current oscillation period $T$ is obtained, the parasitic capacitance value of the PV panel $C_{\mathrm{PV}}$ can be calculated.

\section{Simulation Results}

In order to verify the relationship between the $T$ and the 
TABLE II

Key PARAMETers

\begin{tabular}{lc}
\hline \hline Parameter & Value \\
\hline Input DC Voltage $V_{D C}$ & $18 \mathrm{~V}$ \\
Filter Inductance $1 L_{1}$ & $1 \mathrm{mH}$ \\
Filter Inductance $2 L_{2}$ & $1 \mathrm{mH}$ \\
Filter Capacitance $C$ & $25 \mu \mathrm{F}$ \\
Load Resistance $R$ & $5 \Omega$ \\
Photovoltaic panel parasitic capacitance $C_{\mathrm{PV}}$ & $5 \mu \mathrm{F}$ \\
\hline \hline
\end{tabular}

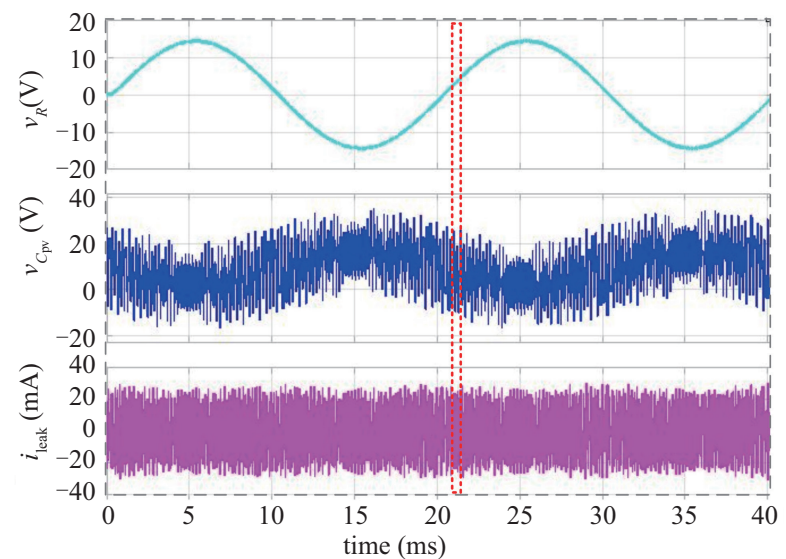

(a)

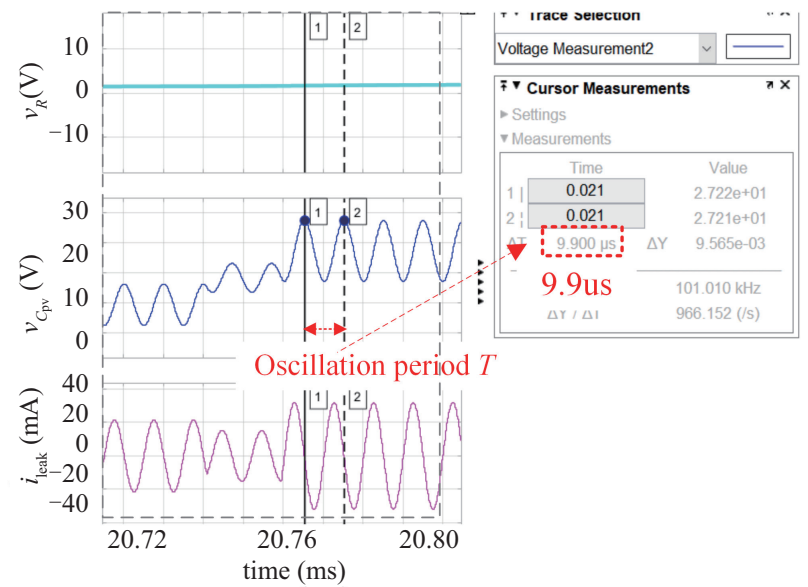

(b)

Fig. 7. (a) The main simulation waveforms when $C_{\mathrm{PV}}$ is $5 \mathrm{nF}$. (b) Zoom in the main waveforms to get the oscillation period.

$C_{\mathrm{PV}}$, a single-phase full-bridge simulation circuit is built. In the simulation, a certain value $C_{\mathrm{PV}}$ is given to measure the $T$. The key simulation parameters are listed in Table II.

When the value of $C_{\mathrm{PV}}$ is $5 \mathrm{nF}$, the main waveforms are shown in Fig. 7, in which, $v_{R}$ is the voltage of the load resistance, $v_{\mathrm{Cpv}}$ is the voltage of the $C_{\mathrm{Pv}}$ and $i_{\text {leak }}$ is the current of $C_{\mathrm{PV}}$.

It can obtain the leakage current oscillation period value $T$ of the simulation waveforms is 9.9 us when the $C_{\mathrm{PV}}$ is $5 \mathrm{nF}$. Then,

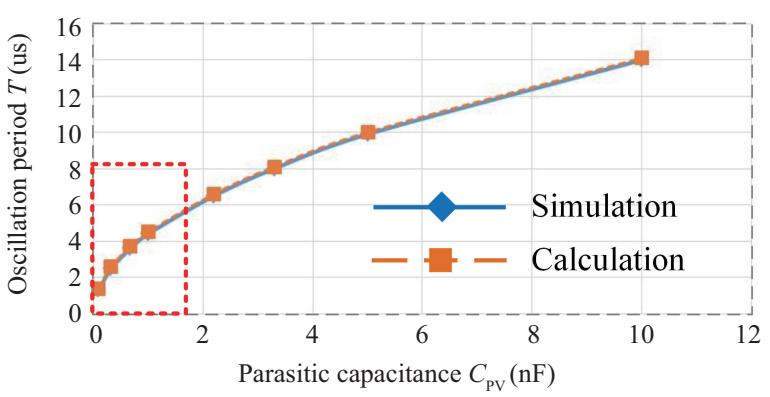

(a)

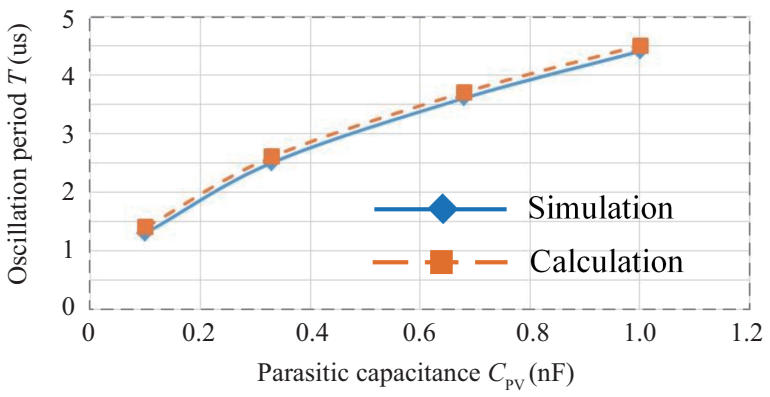

(b)

Fig. 8. (a) Simulation and calculation of the relationship between the parasitic capacitance value and the oscillation period. (b) Zoom in on the part of (a).

under the other parameters of simulation, $T$ is brought into (3) and calculated the value of $C_{\mathrm{PV}}$ is about $5 \mathrm{nF}$.

In order to verify the relationship between the $C_{\mathrm{PV}}$ and $T$, the simulation is carried out under different $C_{\mathrm{PV}}$. Fig. 8 shows the comparison between the simulated values and the calculated values by (3). It can get that the $T$ obtained by circuit simulation and theoretical analysis is basically the same under the different $C_{\mathrm{PV}}$ values.

\section{EXPERIMENTAL RESULTS}

In order to verify the correctness of the proposed method and accurately obtain the value of $C_{\mathrm{PV}}$, the following experiments were carried out. First, an actual capacitor is connected to the DC source to simulate the PV panel to do the experiment. Then experiment with the actual PV panel, which type is SFPVM-50. Finally, the impedance analyzer is used to measure the $C_{\mathrm{PV}}$ of this actual PV panel under the same environment as the circuit experiment.

The single-phase full-bridge inverter experimental platform is shown in Fig. 9. The values of the DC voltage, filter inductance, filter capacitance, and the load are the same as those in Table II. The MOSFET used in the experiment inverter is IRF540NPbF. The inverter uses unipolar \& frequency multiplication SPWM modulation with a switching frequency of $5 \mathrm{kHz}$. The leakage current is picked up by Tektronix TCP305 current probe and then analyzed by Tektronix MDO324 oscilloscope to get the time-domain waveform oscillation period and the Fast Fourier Transform (FFT) results. 


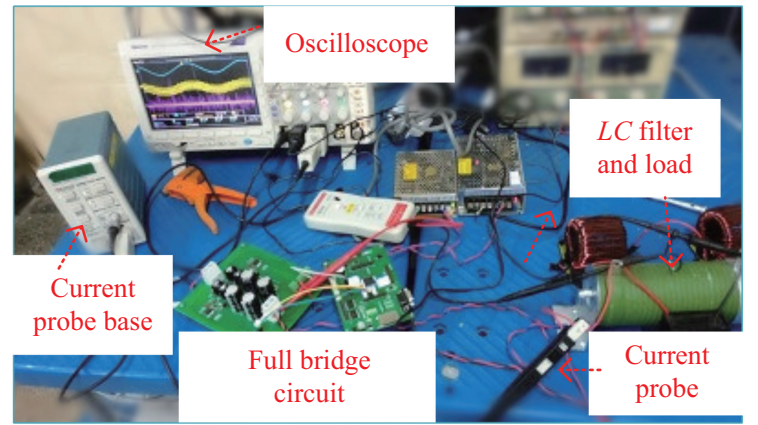

Fig. 9. Single-phase full-bridge inverter experimental platform.

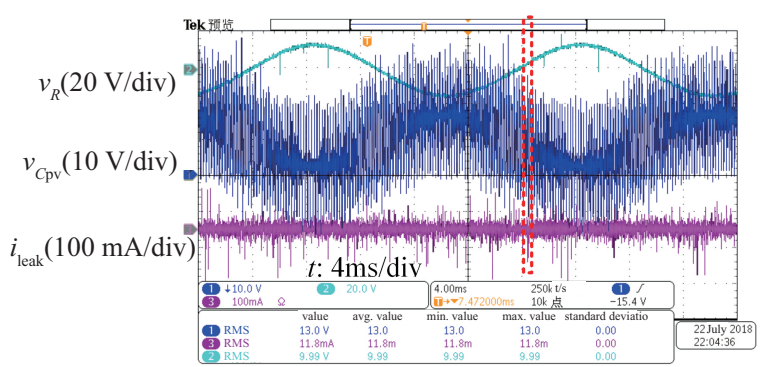

(a)

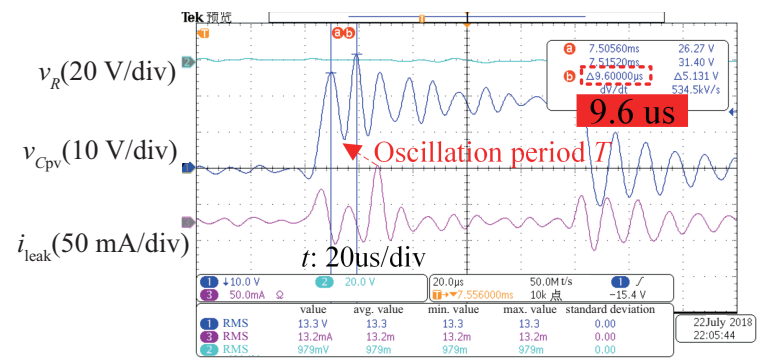

(b)

Fig. 10. (a) The main time-domain experimental waveforms for DC source circuit verification. (b) Zoom in on the main waveforms to get the oscillation period.

\section{A. DC Source Experimental Verification of Oscillation Method}

An actual $5 \mathrm{nF}$ capacitance is connected to the $18 \mathrm{~V} \mathrm{DC}$ source to simulate the PV panel to do the experiment. Fig. 10 shows the main time-domain experimental waveforms under the same parameters of the simulation above. Compared with the simulation, the parameters such as the actual device are not ideal, which results in the difference between the actual circuit waveforms and the simulation waveforms. The parasitic resistance in the loop consumes energy, causing the oscillation of the actual circuit is attenuation oscillation, but not affect the oscillation period of leakage current.

In order to observe the resonant frequency point more conveniently, the time-domain waveform of leakage current is analyzed by FFT, and the result is shown in Fig. 11. Due to the noise energy of the inverter is mainly concentrated near the switching frequency of integer multiples, the leakage current will have a series of peaks at the corresponding frequency point. However, At the resonant frequency point, the peak is more obvious because of the smallest impedance of the circuit

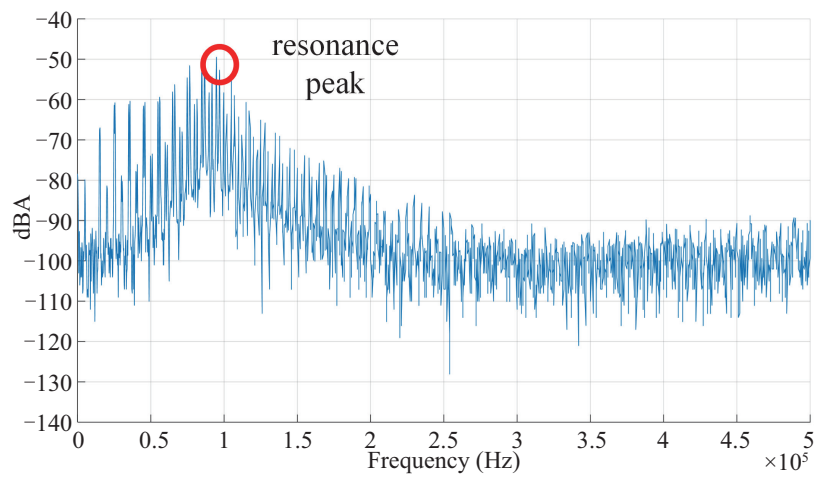

Fig. 11. Leakage current spectrum distribution map.

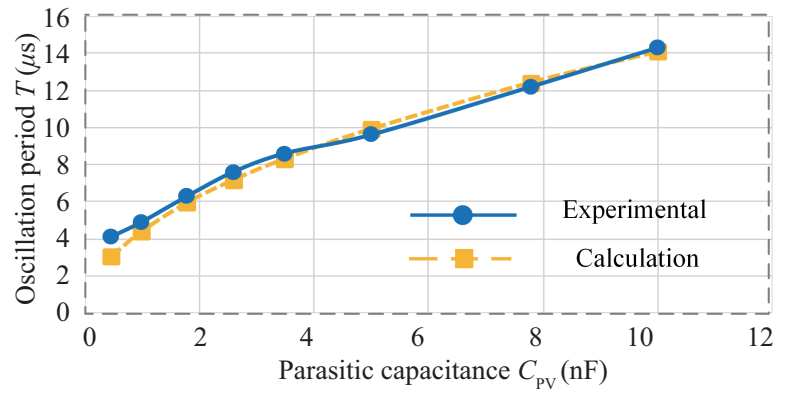

Fig. 12. Experimental and calculation of the relationship between the parasitic capacitance value and the oscillation period.

[26]. The amplitude of the leakage current harmonic at 100 $\mathrm{kHz}$ is the largest, and the system has a resonant peak near this frequency. The oscillation periods obtained in the time-domain and the frequency-domain are basically the same.

From the experimental time-domain waveforms and the spectral distribution, the leakage current oscillation period of the actual capacitance of $5 \mathrm{nF}$ is about 9.6 us. Bring this oscillation period $T$ into (3), and the calculated $C_{\mathrm{PV}}$ is 4.67 $\mathrm{nF}$. The experimental result error is $6.6 \%$, which is within the acceptable error range.

The reasons for the error may be:

1) The actual capacitance used and the filter inductances are not standard;

2) Errors caused by the experimental process;

3) Simplification in theoretical analysis.

In order to verify the method proposed in this paper, the experimental is carried out under different $C_{\mathrm{PV}}$. Fig. 12 shows the comparison between the experimented values and the calculated values by (3). The results show that the $T$ values obtained from the circuit experiment and theoretical analysis are very close at different $C_{\mathrm{PV}}$ values. When the $C_{\mathrm{PV}}$ is Picofarad level, the experimental value is larger than the calculated value, because other parasitic parameters in the circuit, such as the parasitic capacitances between the switchto- heatsink/ground, cannot be ignored directly. However in the actual PV plant, the $C_{\mathrm{PV}}$ vary from $7 \mathrm{nF} / \mathrm{kW}[11]-[12]$ to $220 \mathrm{nF} / \mathrm{kW}$ [13]-[14], the method proposed in this paper can accurately extract the $C_{\mathrm{PV}}$ of the actual PV plant. 


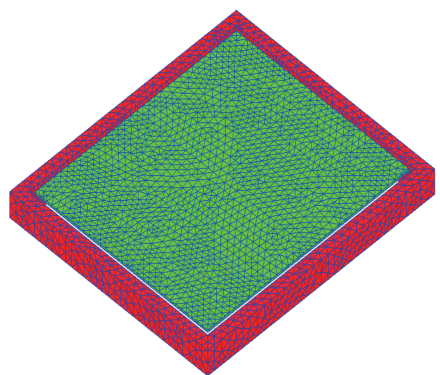

(a)

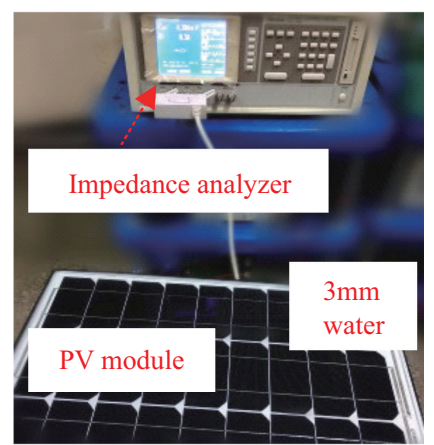

(b)

Fig. 13. (a) 3D FE simulation method. (b) Impedance analyzer measurement platform.

\section{B. Comparison of Photovoltaic Panel Parasitic Capacitance Extraction Methods}

Because the $C_{\mathrm{PV}}$ will increase dramatically in a rainy environment [2]-[4], which has a great influence on the leakage current of the non-isolated system. It is important to extract the capacitance value accurately in a damp environment, so researchers pay more attention to $C_{\mathrm{PV}}$ in a rainy environment. This part compared with the on-line extraction method proposed in this paper with 3D FE simulation, the measurement by impedance analyzer and existing on-line calculation method, which are compared under the same rainy environment. The 3D FE simulation and the measurement through the impedance analyzer platform are shown in Fig. 13. The existing on-line calculation method platform is similar to the experimental platform of this paper, which is shown in Fig. 9.

According to the size of the actual PV panel, the PV model in the rainy environment is established in Ansys Maxwell, and the value of $C_{\mathrm{PV}}$ is obtained by simulation. The method of impedance analyzer measurement picks up the $C_{\mathrm{PV}}$ by Chroma 3302. Simulating the PV panel in the rainy environment by adding water to the PV panel. During the experiment, it was found that when the thickness of the water added to the PV was about 3 millimeters, the value of $C_{\mathrm{PV}}$ reached the maximum, sothe PV in the experiment was carried out after adding water of 3 millimeters.

In the same rainy environment, an actual $18 \mathrm{~V}$ PV panel to do the experiment. the period in the on-line oscillation period method proposed in this paper can be obtained from the timedomain or frequency-domain of the leakage current. Fig. 14 shows the main time-domain experimental waveforms with the

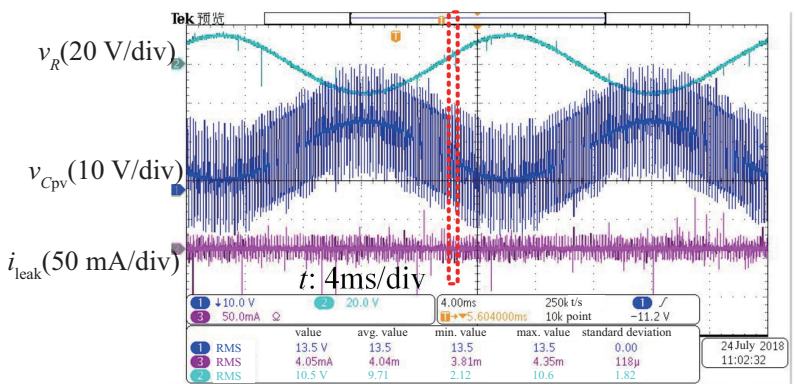

(a)

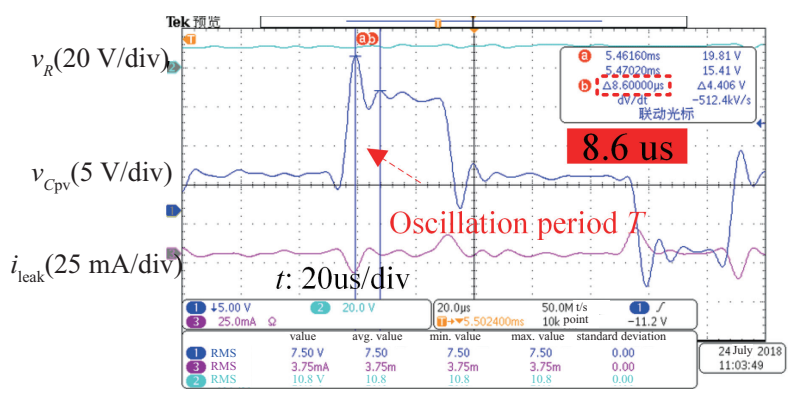

(b)

Fig. 14. (a) The main time-domain experimental waveforms for the PV panel in the rainy environment. (b) Zoom in on the main waveforms to get the oscillation period.

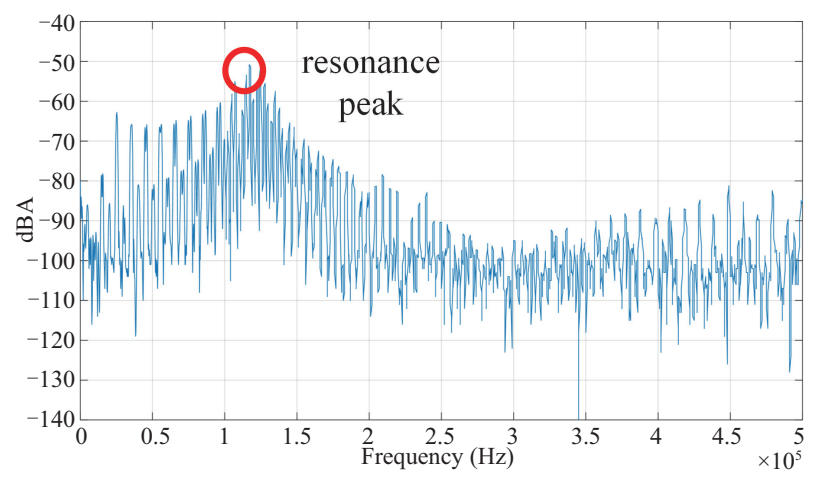

Fig. 15. Leakage current spectrum distribution map in the rainy environment.

PV panel in the rainy environment. Fig. 15 shows the spectrum distribution of the leakage current in the rainy environment.

The parasitic resistance in the loop consumes energy, causing the oscillation of the actual circuit is attenuation oscillation. The amplitude of the leakage current harmonic at $115 \mathrm{kHz}$ is the largest, and the system has a resonant peak near this frequency. From the time-domain experimental waveforms and the resonant peak of the spectrum distribution, the leakage current oscillation period $T$ is 8.6 us, which is brought to the (3), and the calculated $C_{\mathrm{PV}}$ is $3.75 \mathrm{nF}$.

According to the existing on-line calculation method by reference [7], Table III shows the main components of voltage and current on the capacitance.

The $C_{\mathrm{PV}}$ value $3.21 \mathrm{nF}$ was obtained by average of the series of calculated capacitance values in Table III. The results of the $C_{\mathrm{PV}}$ values of these four extracted are shown in Table IV.

The parasitic capacitance values extracted by these four 
TABLE III

The Existing ON-Link Calculation Method

\begin{tabular}{cccc}
\hline \hline $\begin{array}{c}\text { Harmonic } \\
\text { frequency } \\
f(\mathrm{kHz})\end{array}$ & $\begin{array}{c}\text { Voltage on the } \\
\text { capacitance } \\
V_{C \mathrm{p}}(\mathrm{V})\end{array}$ & $\begin{array}{c}\text { Current on the } \\
\text { capacitance } \\
I_{\mathrm{Cp}}(\mathrm{mA})\end{array}$ & $\begin{array}{c}\text { Capacitance } \\
(\mathrm{nF})\end{array}$ \\
\hline 5 & 5.4 & 0.6 & 3.54 \\
15 & 1.2 & 0.37 & 3.27 \\
25 & 0.6 & 0.32 & 3.39 \\
35 & 0.4 & 0.3 & 3.41 \\
45 & 0.34 & 0.32 & 3.33 \\
55 & 0.3 & 0.35 & 3.38 \\
65 & 0.27 & 0.36 & 3.26 \\
75 & 0.28 & 0.41 & 3.11 \\
85 & 0.29 & 0.51 & 3.29 \\
95 & 0.37 & 0.68 & 3.08 \\
105 & 0.5 & 0.98 & 2.97 \\
115 & 0.67 & 1.3 & 2.95 \\
125 & 0.42 & 0.94 & 2.85 \\
\hline \hline
\end{tabular}

TABLE IV

Photovoltaic Parastitic Capacttance Values Obtained Under Different MEASUREMENT Modes

\begin{tabular}{lc}
\hline \hline Measurement method & Parasitic capacitance $C_{\mathrm{PV}}$ \\
\hline 3D finite element simulation & $3.50 \mathrm{nF}$ \\
Impedance analyzer test & $3.85 \mathrm{nF}$ \\
Circuit test of oscillation & $3.75 \mathrm{nF}$ \\
period in this paper & \\
The existing on-line calculation & $3.21 \mathrm{nF}$ \\
method & \\
\hline \hline
\end{tabular}

methods are close to each other. The method of the numerical simulation depends on the accurate establishment of the model. Although the direct method can be measured accurately, the off-line method is not suitable for the actual variable environment. The existing on-line calculation method does not take into account the effects of parasitic resistance of the PV panels, resulting in inaccurate results. From the above experiments, the on-line method proposed in this paper to extract the $C_{\mathrm{PV}}$ based on the leakage current oscillation period can accurately extract the value of $C_{\mathrm{PV}}$.

\section{CONCLUSIONS}

This paper analyzes the switching processes of the singlephase full-bridge non-isolated inverter. Through these switching processes, a simplified resonant model is obtained. And the relationship between parasitic capacitance and leakage current oscillation period is derived. Through the analysis of the leakage current oscillation mechanism and the limitations of the existing PV panel parasitic capacitance extraction methods, this paper proposes an on-line method suitable for the actual running environment. After theoretical analysis and simulations, then carries out experimental verification. The measurement is convenient and accurate. Finally, in the rainwater environment, this method is compared with the extraction method of 3D FE simulation, impedance analyzer test and the existing on-line calculation method, the accuracy of this method is verified.

\section{REFERENCES}

[1] J. Wang, Y. Xun, and S. Yu, "An extraction method for the parasitic capacitance of the photovoltaic module based on the oscillation of the leakage current," 2019 IEEE 10th International Symposium on Power Electronics for Distributed Generation Systems (PEDG), Xi'an, China, 2019, pp. 945-950.

[2] W. Chen, X. Yang, W. Zhang and X. Song, "Leakage current calculation for PV inverter system based on a parasitic capacitor model," in IEEE Transactions on Power Electronics, vol. 31, no. 12, pp. 8205-8217, Dec. 2016.

[3] S. Yu, J. Wang, X. Zhang, and F. Li, "Complete parasitic capacitance model of photovoltaic panel considering the rain water," in Chinese Journal of Electrical Engineering, vol. 3, no. 3, pp. 77-84, Dec. 2017.

[4] S. Yu, J. Wang, and X. Zhang, "Effect of water on parasitic capacitance of photovoltaic panel," in IEEE 2017 Energy Conversion Congress and Exposition Conference, 2017, pp. 4414-4419.

[5] H. Xiao and S. Xie, "Leakage current analytical model and application in single-phase transformerless photovoltaic grid-connected," in IEEE Transactions on Electromagnetic Compatibility, vol. 52, no. 4, pp. 902913, Nov. 2010.

[6] J. Myrzik and M. Calais, "String and panel integrated inverters for single-phase grid connected photovoltaic systems A review," in IEEE 2003 Bologna Power Tech Conference, 2003, pp. 430-437.

[7] O. López, F. Freijedo, and A. Yepes, "Eliminating ground current in a transformerless photovoltaic application," in IEEE Transactions on Energy Conversion, vol. 25, no. 1, pp. 140-147, Mar. 2010.

[8] W. Li, Y. Gu, H. Luo, W. Cui, X. He and C. Xia, "Topology review and derivation methodology of single-phase transformerless photovoltaic inverters for leakage current suppression," in IEEE Transactions on Industrial Electronics, vol. 62, no. 7, pp. 4537-4551, July 2015.

[9] O. Lopez, R. Teodorescu, and J. Doval-Gandoy, "Multilevel transformerless topologies for single-phase grid-connected converters," IECON 2006 - 32nd Annual Conference on IEEE Industrial Electronics, Paris, 2006, pp. 5191-5196.

[10] B. Yang, W. Li, Y. Gu, W. Cui, and X. He, "Improved transformerless inverter with common-mode leakage current elimination for a photovoltaic grid-connected power system," in IEEE Transactions on Power Electronics, vol. 27, no. 2, pp. 752-762, Feb. 2012.

[11] W. Cha, K. Kim, Y. Cho, S. Lee, and B. Kwon, "Evaluation and analysis of transformerless photovoltaic inverter topology for efficiency improvement and reduction of leakage current," in IET Power Electronics, vol. 8, no. 2, pp. 255-267, 22015.

[12] J. Zhang, W. Chen, B. Zhang, X. Song, and H. Huang, "Optimal design of EMI filters for PV system based on parasitic parameter and stability analysis," 2015 9th International Conference on Power Electronics and ECCE Asia (ICPE-ECCE Asia), Seoul, 2015, pp. 2744-2751.

[13] X. Guo, R. He, J. Jian, Z. Lu, X. Sun, and J. M. Guerrero, "Leakage current elimination of four-leg inverter for transformerless three-phase PV systems," in IEEE Transactions on Power Electronics, vol. 31, no. 3, pp. 1841-1846, March 2016.

[14] M. C. Cavalcanti, K. C. de Oliveira, A. M. de Farias, F. A. S. Neves, G. M. S. Azevedo, and F. C. Camboim, "Modulation techniques to eliminate leakage currents in transformerless three-phase photovoltaic systems," in IEEE Transactions on Industrial Electronics, vol. 57, no. 4, pp. 13601368, Apr. 2010.

[15] Rodolfo Araneo, Sergio Lammens, Marco Grossi, and Stefano Bertone. "EMC issues in high-power grid-connected photovoltaic plants", in IEEE Tansactions on Electromagnitics Compatibility, vol. 51, no. 3, 
Aug. 2009, pp. 639-648.

[16] S. B. Kjaer, J. K. Pedersen, and F. Blaabjerg, "A review of single-phase grid-connected inverters for photovoltaic panels," in IEEE Transactions on Industry Applications, vol. 41, no. 5, pp. 1292-1306, Sept.-Oct. 2005.

[17] S. Oh and H.-S. Philip Wong, "Physics-based compact model for III$\mathrm{V}$ digital logic FETs including gate tunneling leakage and parasitic capacitance," in IEEE Transactions on Electron Devices, vol. 58, no. 4, pp. 1068-1075, Apr. 2011.

[18] L. Wei, J. Deng and H.S-.P. Wong, "Modeling and performance comparison of 1-D and 2-D devices including parasitic gate capaci-tance and screening effect," in IEEE Transactions on Nanotechnology, vol. 7, no. 6 , pp. 720-727, Nov. 2008.

[19] Wenjie Chen, Xiaomei Song, Hao Huang, and Xu Yang, "Numerical and experimental investigation of parasitic edge capacitance for photovoltaic panel," 2014 International Power Electronics Conference (IPECHiroshima 2014 - ECCE ASIA), Hiroshima, 2014, pp. 2967-2971.

[20] Z. Liu, X. Huang, and F. Lee, "Package parasitic inductance extraction and simulation model development for the high-voltage cascode GaN HEMT," in IEEE Transactions on Power Electronics, vol. 29, no. 4, pp. 1977-1985, 2014.

[21] T. Liu, T. Wong, and S. John, "A new characterization technique for extracting parasitic inductances of $\mathrm{SiC}$ power MOSFETs in discrete and panel packages based on two-port S-parameters measurement," in IEEE Transactions on Power Electronics, vol. 33, no. 11, pp. 98199833, Nov. 2018

[22] X. Zhang, Solar Photovoltaic Grid-connected Power Generation and Its Inverter Control, Mechanical Industry Press, Beijing, 2011, pp.97103.

[23] P. Zhang, Q. Wan, C. Feng, and H. Wang, "All regimes parasitic capacitances extraction using a multi-channel CBCM technique," in IEEE Transactions on Semiconductor Manufacturing, vol. 30, no. 2, pp. 121-125, May 2017.

[24] E. Gubia, P. Sanchis, A. Ursua, J. Lopez, and L. Marroyo, "Ground current in single-phase transformerless photovoltaic systems," in Progress in Photovoltaics: Research and Applications, New York: Wiley, pp. 629-650, 2007.

[25] Y. Chen, D. Xu, and J. Xi, "Common-mode filter design for a transformerless ZVS full-bridge inverter," in IEEE Journal of Emerging and Selected Topics in Power Electronics, vol. 4, no. 2, pp. 405-413, June 2016.

[26] V. Sonti, S. Jain, and S. Bhattacharya, "Analysis of the modulation strategy for the minimization of the leakage current in the PV gridconnected cascaded multilevel inverter," in IEEE Transactions on Power Electronics, vol. 32, no. 2, pp. 1156-1169, Feb. 2017.

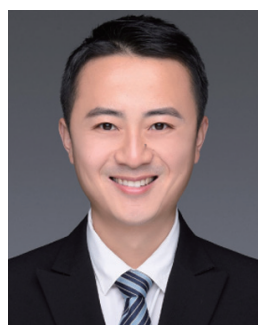

Jianing Wang was born in Anhui, China. He received the M.S. degree in Power Electronics from Power Electronics and Renewable Energy Center in Xi'an Jiaotong University, Xi'an, China, in 2010, and received the Ph.D. degree in the Electrical Power Processing Group (EPP), Faculty of Electrical Engineering, Delft University of Technology, Delft, The Netherlands, in 2014. At the end of 2014 he became an associate professor at Hefei University of Technology, Hefei, China. He was the deputy secretary of ECCE ASIA 2016, Hefei, and became a member of China Power Supply Society (CPSS) since then. He was also the associate editor of the Chinese Journal of Electrical Engineering since 2016. His current research interests include the integration of power electronics converters, the application of wide bandgap power devices, and the analysis of parasitics in converters and PV modules.

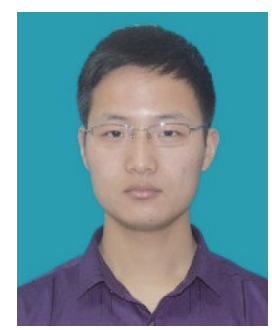

Yuanwu Xun was born in Anhui, China. He received the B.S. degree in Electrical Engineering in Anhui Agricultural University, Hefei, China, 2017. $\mathrm{He}$ is currently working toward the M.S. degree at the Department of Electrical Engineering and Automation in Hefei University of Technology.

His current research interests include the parasitics of the photovoltaic modules, the application of wide bandgap power devices, and soft-switching

application in inverters.

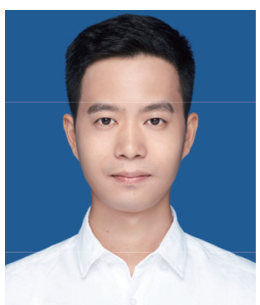

Xiaohui Liu was born in Anhui, China, in 1990. He received the B.S. degree in Electrical Engineering and Automation in Hefei University of Technology, Hefei, China, 2011. He is currently working toward the M.S. degree at the Department of Electrical Engineering and Automation in Hefei University of Technology.

His current research interests include common the wide bandgap devices. current elimination and the parasitic parameters of

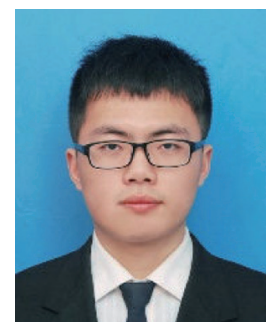

Shaolin Yu received the B.S. degree in Electrical Engineering from the School of Electrical Engineering and Automation, Hefei University of Technology, Hefei, China, in 2015, and the M.S. degree in Control Engineering in 2018 from the School of Electrical Engineering and Automation, Hefei University of Technology, Hefei, China, where he is currently working toward the Ph.D. degree at the Department of Electrical Engineering and Automation.

His current research interests include modeling of parasitic parameter in converters and power electronics applications for wide bandgap devices. 